\title{
Blended Learning 2.0 con Mundos Virtuales
}

Calixto Alejandro Maldonado ${ }^{1}$ y Patricia Etcheverry ${ }^{1}$

\section{Resumen}

El presente artículo contiene una propuesta de aplicación de Mundos Virtuales (MV) basada en las teorías más actuales sobre aprendizaje y aportes de la didáctica situada y en contexto, y todo ello asociado a estrategias que utilicen TICs, como es el caso de Blended Learning (BL). Proponemos, aplicando prácticas de Ingeniería de Software, agregarle a BL otro componente tecnológico, como es el de MV junto a los recaudos antes mencionados, a fin de promover aprendizaje amigable y significativo en los estudiantes, mayoritariamente nativos digitales y además direccionar hacia aquellos con discapacidad motora a fin de que les sea posible completar la etapa presencial del BL de base. A esta estrategia de mejora representada en una nueva versión del modelo BL ahora vinculada a MV la denominamos "Blended Learning 2.0".

Palabras Clave: Mundos Virtuales; Aprendizaje Colaborativo; Blended Learning; Requerimientos de Software. 


\section{Abstract}

This paper contains a proposal of Virtual World (VW) application based on current theories about learning and contributions to situated and context teaching, and all associated with strategies using ICT, such as Blended Learning (BL). We propose, applying Software Engineering practices, to add BL another technological component, as VW with the abovementioned precautions, to promote friendly and meaningful learning in students, mainly digital natives, and also address to those with disabilities so that they can complete BL in-person class. This improvement strategy represented in a remake of the BL model now linked to VW is called "Blended Learning 2.0."

Keywords: Virtual Worlds, Collaborative Learning, Blended Learning, Software Requirements. 


\section{Introducción.}

La propuesta se vincula con la práctica de Ingeniería de Software que permita crear una aplicación con componentes visuales dentro de un Mundo Virtual (MV), y simultáneamente que soporte actividades didácticas para mejorar el aprendizaje de estudiantes de las carreras de Informática.

La implementación de un estudio sistemático (Pérez Cota, 2012) realizado sobre la factibilidad de alternativas didácticas, ha permitido definir los requerimientos que mejor sustentan las estrategias en las actividades con software y hardware de Mundos Virtuales.

Los requerimientos didácticos han sido relevados a partir de trabajos de investigación cualitativa en dos Universidades y en cinco Colegios de Nivel Medio de Argentina. A lo que se suma un estudio del software Second Life (SL), que evidenció su funcionamiento en coordinación con MOODLE, es decir en asociación con un sistema de administración de aprendizaje o LMS -por sus siglas en inglés de Learning Management Systems - que implica procedimientos de integración entre estas dos plataformas, a la que se denomina SLOODLE. Asimismo se presenta una propuesta integradora con un ejemplo de actividad pedagógica situada realizada en el entorno de MV. En este caso se analizaron los lenguajes de programación LSL de SL y PHP para Moodle.

\section{Marco teórico y Requerimientos Didácticos.}

El escenario socio-cultural que inaugura la Era Digital produce nuevos hábitos, valores y costumbres que en este artículo son tomados en cuenta a fin de definir requerimientos de la didáctica actual en asociación con la aplicación de herramientas TICs en el área de educación. El propósito es atender eficazmente la oportunidad de aplicación de TICs en los procesos de Enseñanza y Aprendizaje de los estudiantes que asisten a la Universidad en carreras vinculadas a la informática.

La didáctica actual viene enunciando el Paradigma Constructivista (Carretero, 2009) como la llave de una genuina apropiación de conocimiento. O dicho de otro modo, la internalización de los contenidos de la enseñanza con posibilidad de evocación y efectiva recordación. En este sentido la Didáctica actual se reconoce a través de la construcción teórica de autores pilares de esta línea de pensamiento.

\subsection{Fundamento Teórico del Paradigma Constructivista}

La teoría formulada por Jean Piaget acerca de la relación entre alcance madurativo y procesos cognitivos marca el surgimiento del Paradigma Constructivista del Conocimiento, línea de investigación seguida por especialistas en la materia como Ausubel, Bruner, Vigotsky, Carretero, Litwin y Souto (1999), entre otros. 
En su afán por explicar esta lógica recursiva y abarcativa, Piaget desarrolló los conceptos de asimilación, acomodación y equilibrio como actividades activas del sujeto, en donde la sucesión, inclusión y re significación de las etapas sucesivas, culminarían en la mejor adaptación y ubicación intelectiva del sujeto en relación al medio y su influencia. En este sentido, Piaget sostiene un constructivismo basado en la actividad adaptativa y operatoria de la inteligencia de naturaleza auto estructurante, es decir frente al conflicto cognitivo por efecto del medio el sujeto se transforma, Línea teórica que entre otras cosas refuta el acto del conocer como mera copia - o mecanicismo - de la realidad.

Desde una posición más actual del constructivismo, Jerome Bruner plantea en "La educación puerta de la cultura" (Bruner, 1997) el plus que aporta a la estrategia didáctica el aprendizaje por descubrimiento, junto con el aporte fundamental de la influencia de la imagen y la narrativa icónica, en el acto mismo de conocer.

En cuanto a la imagen, los estudios de Bruner acuerdan en que el ícono gráfico repercute de un modo singular en la mente del estudiante -además de resultar amigable y atractivo- impidiendo el juicio crítico, y en consecuencia incorporando el contenido por sugestión, con lo cual la imagen se ubica como soporte y nuevo lenguaje de código propio.

En otra línea, David Ausubel (1983) sostiene la importancia de conocer la estructura cognitiva del estudiante en términos de conocimientos previos y el modo singular en que se organiza. Este autor ofrece el marco para el diseño de herramientas meta cognitivas que permitirían conocer semántica y formalmente las organizaciones singulares de los estudiantes, posibilitando de este modo una mejor orientación en la labor educativa. Ausubel resume este hecho en el epígrafe de su obra de la siguiente manera: "Si tuviese que reducir toda la psicología educativa a un solo principio, enunciaría este: el factor más importante que influye en el aprendizaje es lo que el alumno ya sabe. Averígüese esto y enséñese consecuentemente" (Ausubel, 1983).

Desde esta posición puede interpretarse que el profesor respecto de su grupo clase puede enfrentarse con, por lo menos, dos cuestiones. Por un lado y en cuanto al contenido, la incertidumbre acerca de los saberes previos de su grupo, es decir, aquellos saberes ya internalizados en sus estudiantes que le permitirían a modo de anclaje sostener aquellos que vendrán vehiculizados a través de actividades didácticas y situaciones de aprendizaje nuevas. La segunda cuestión se refiere a la incertidumbre acerca del nivel cognitivo-madurativo alcanzado por los estudiantes que, de aclararse permitiría la toma de decisión y puesta en práctica de líneas de acción didácticas bien precisas y funcionales al grupo y así ajustar acertadamente la didáctica aplicada a fin de promover verdadero conocimiento.

Esta posibilidad diagnóstica posibilitaría la construcción de "organizadores previos" en función de garantizar código compartido y activar esquemas relacionales 
que garanticen la significatividad de los contenidos impartidos y al mismo tiempo el pasaje hacia la retención de los conceptos.

Por su parte, el aporte de Lev Semiónovich Vigotsky, el autor de Zona de Desarrollo Próximo (1988), se centra básicamente en la influencia social en la construcción de conocimiento. Para este autor, los Procesos Psicológicos Superiores, con fundamento en la interacción, la apropiación y la internalización de los contenidos de enseñanza se presentan siempre mediatizados desde un contexto social e histórico determinado. La teoría Socio-cultural de Vigotsky refiere a que el conocimiento provendría de lo "inter-subjetivo" o contextual -el medio social-, siempre mediatizado por actividades que posibilitaría la apropiación de la cultura, y desde allí migrar hacia lo "intra-subjetivo" o psicológico. Jerarquizando de ese modo las actividades en el marco del desenvolvimiento social y la colaboración entre pares. Es decir, entre todos se aprende más y mejor.

En el contexto de las Tecnologías de la Información y la Comunicación (TICs) el aprendizaje colaborativo, ha tomado protagonismo, en la medida de la existencia de flujos de significación en retroalimentación continua y permanente, potenciado justamente por las Nuevas Tecnologías. Es decir, en el marco de la Sociedad del Conocimiento y el Conocimiento Compartido, el aprendizaje colaborativo con apoyo en las TICs refuerza un sistema de comunicación abierto y horizontal creando claramente nuevas situaciones de aprendizaje.

\subsection{Evidencia en las Nuevas Competencias de los Estudiantes}

En la actualidad existen acuerdos respecto del diferencial asignado a las aptitudes de los Nativos Digitales (Prensky, 2006) y (Piscitelli, 2009) reconociendo en ellos a los estudiantes actuales y a los futuros.

Marc Prensky basa sus presunciones en la estructura mental de los jóvenes educados en un mundo digital, y sostiene que la misma es diferente a la de los educados en décadas anteriores. Describe que los jóvenes han pasado más horas frente a la televisión y los juegos que las que han dedicado a la lectura y esto, junto con la afirmación acerca de que la experiencia influye en la estructura mental, deriva necesariamente en el postulado acerca de que a estructuras mentales de topología diferente, le corresponderían diferentes canales de comunicación a fin de interesarlos como sujetos de aprendizaje (Prensky, 2006).

Desde la simple observación resulta obvia la excelente motivación e interés de los jóvenes frente a los juegos interactivos.

En definitiva, se plantea la posibilidad de aprovechar esta atracción a los juego interactivos a fin de sumar valor a las situaciones de enseñanza y aprendizaje que de por sí deben ser consideradas en función de los requerimientos didácticos y a la vez facilitar el acceso a personas que por impedimentos físicos puedan participar de actividades originalmente previstas para ser físicamente presenciales. Asimismo, 
para los autores resulta de suma importancia el uso de MV en una instalación de acceso público como por ejemplo, Second Life para funcionar en la elaboración de actividades que resulten claramente significativas para el aprendizaje.

\section{Requerimientos didácticos asociados al software de MV}

La influencia del Paradigma Constructivista en el Desarrollo del Blended Learning 2.0 (con MV) determina los siguientes requerimientos:

Sobre la creación de una actividad lúdica que contenga el tema próximo a estudiar:

- Es prioridad seleccionar un contenido curricular del prescripto, una unidad conceptual con corte temático, que posibilite la construcción con lógica interna, de una actividad con un formato de juego grupal del tipo por ejemplo, de una competencia que posibilite la detección diagnóstica de los saberes previos y el estadio cognitivo de los estudiantes a fin de permitirle al docente el armado de los organizadores previos, es decir, el constructo de puentes cognitivos.

Sobre la estructura de la actividad:

- Las actividades en MV, ya sean inmersivas o de juego interactivo, sería óptimo que contemplen una fase introductoria a modo de ejemplo corto, siempre visual, gráfico o animado, a fin de inducir sugestivamente y de modo amigable y motivador al estudiante.

- El modelo de juego interactivo debiera contener situaciones problemáticas o dilemáticas promoviendo de este modo conflicto cognitivo y desequilibrio provocando así nuevas acomodaciones adaptativas y avance cualitativo del pensamiento, es decir, generar aprendizaje

- La actividad dentro del mundo virtual debiera permitir usar una aplicación de chat, permitiendo así el aprendizaje colaborativo entre grupos de pares. Es decir, resulta esencial plantear actividades colaborativas que garanticen resultados positivos de internalización y apropiación de conocimiento. Por ejemplo, registrar vía chat los distintos niveles de los contenidos trabajados en la elaboración discursiva de la actividad. En el caso de un juego con postas o desafíos a modo de diálogo construir colaborativamente la síntesis del trabajo. Permitiendo el chequeo en espiral dialéctico y el ajuste de las variaciones surgidas y su verificación entre todos los participantes, creando así un verdadero ambiente de aprendizaje

\subsection{Aspectos Técnicos del software de MV}

Second Life es un sitio web y servidor de MV lanzado el 23 de junio de 2003, desarrollado por Linden Lab, accesible de modo gratuito para transitar por él. Es pago si se renta una porción de terreno donde instalar los activos, 
en forma de construcciones de diversas texturas, anuncios, habitaciones, salones, auditorios, etc. Los usuarios que abonan por su "tierra", conocidos como residentes, guardan los objetos que encuentran en este mundo virtual dentro de un "inventario" que reúne todo de lo que puede disponer y utilizar (Second life, 2013).

Los usuarios ejecutan en sus equipos un programa de interfaz llamado viewer o visor. Con este visor, el mundo virtual envía la visualización del entorno y también el usuario remite la información de sus movimientos, a fin de que la visualización los refleje. Este mecanismo permite transitar por el MV e interactuar con otros usuarios mediante un avatar (Campazzo, 2011). Es una plataforma que puede ser utilizada para numerosas actividades entre las que se destaca una importante comunidad de Juegos de Roles.

SL tiene dos características que lo diferencian del resto de mundos virtuales, la primera es ofrecer absoluta libertad a sus residentes para lo que puedan realizar, es decir, que no existen instrucciones ni consignas a seguir como sí en los juegos de roles dentro de SL. La segunda característica distintiva es la posibilidad de tener rentados lugares para construir, habitar y recibir visitas de otros avatares pagando una licencia de acceso con un canon fijo anual o mensual.

Para agregar interactividad y comportamientos a los avatares y dispositivos creados en SL, se utiliza un lenguaje de Scripting llamado LSL por Linden Scripting Language. (LSL, 2013).

Los entornos virtuales de multi usuarios con acceso masivo (MMVEs sigla en inglés de Massively Multi User Virtual Environments), que se caracterizan por atender a muchos usuarios interactuando en gran escala: miles de los usuarios se interconectan entre sí, en tiempo real, de a pares o en grupos. La escala de MMVEs afecta en gran medida la capacidad de respuesta y la consistencia del mundo de juego percibida por los jugadores. Cuantos más usuarios y más acciones sean generadas por el usuario y compartidas, más difícil será para el servidor apoyar las interacciones, y justamente la dificultad radica en que esto no aumenta en forma lineal sino cuadráticamente respecto del número de usuarios. Por todo lo anterior, los MMVEs son diseñados utilizando todo tipo de optimizaciones. Estas optimizaciones obstaculizan la escalabilidad y tolerancia a fallos, convirtiéndolos en equipos difíciles de mantener y evolucionar. En general, ha habido pocas recomendaciones de características de configuración de arquitecturas MMVE que puedan generalizarse, lo que hace que cada aplicación termine siendo específicamente diseñado para sus necesidades únicas. Sin embargo se ha analizado bibliografía que orienta hacia una arquitectura basada en REST que podría soportar los niveles de carga de trabajo que generarían miles de usuarios que interactúen entre sí (Lopes, 2012). 


\section{Blended Learning 2.0 con Mundos Virtuales}

\subsection{Características de Blended Learning (BL).}

La Revista Ibero Americana de Educación define al BL con las siguientes características:

"Es una combinación eficiente de diferentes métodos de enseñanza a través del uso de un entorno virtual de aprendizaje y sesiones presenciales. Esta estrategia permite alcanzar resultados relevantes gracias a algunos aspectos destacables:

- Desarrolla habilidades de disciplina, autocontrol y aprendizaje autónomo.

- Promueve la adquisición de competencias en el uso de aulas virtuales.

- Promueve la interacción con profesores y otros estudiantes.

- Utiliza nuevos modos de interacción tales como chats, foros, etc.

- Optimiza los tiempos y desplazamientos, dejando lo presencial solo para temas y actividades que así que lo requieran.

- Facilita que el aprendizaje se realice al ritmo personal de cada uno.

- Proporciona la bibliografía y materiales de forma dinámica con antelación a las fases presenciales." (La Fuente, 2012)

El término Blended Learning con su traducción al español nos indica que es "Aprendizaje mixto", definiendo dos partes de un todo, una parte que tiene una forma de enseñanza asincrónica (realizada independientemente por cada participante, no simultáneamente) que está soportado por un LMS para acceder al material de lectura, videos, cuestionarios con algún grado de interactividad y una parte sincrónica (resuelta entre todos los participantes en un mismo momento) y otra presencial, una clase cada cierto tiempo, en donde se encuentran cara a cara los participantes y el profesor del curso.

\subsection{Uso de Mundos Virtuales en Blended Learning 2.0.}

La propuesta de este artículo es mejorar BL con MV como soporte de los encuentros sincrónicos, es decir, realizar las sesiones presenciales utilizando el software de MV a fin de que la totalidad de los participantes desde su ubicación, a la misma hora, asistan virtualmente al encuentro llevado a cabo en el servidor de MV, SL en este caso).

Este uso de un MV como un entorno inmersivo permitiría la visualización y comunicación a todos los participantes del curso, estudiantes y profesores, representados cada uno con un avatar, pudiendo a través de la voz o de un chat interno, interactuar, hacer preguntas, realizar actividades, observar animaciones, visitar escenarios realizados y compartidos por el profesor para experimentar, como lugares famosos, maquetas de procesos industriales, fisiológicos, químicos, físicos, realización de actividades simulando un programa de concursos televisivos y lo que pueda construirse como para ser observado en un MV, buscando lograr 
experiencia sin necesidad de trasladarse al lugar real, permitiendo a personas verdaderamente distantes o con impedimentos físicos también tener la posibilidad de asistir virtualmente a las sesiones presenciales.

Por ejemplo, la actividad a desarrollarse en un MV podría ser una simulación de un concurso televisivo con gradas, pantalla gigante, donde podrían proyectarse preguntas con opciones múltiples sobre el tema que comenzaría a dictarse, para que los participantes pulsen en un control asignado a cada uno de ellos la letra de la respuesta que respondería a las preguntas sobre el nuevo tema que iría apareciendo. Sobre un tablero de control se confirma que cada participante ha respondido y si efectivamente la respuesta es la correcta. La información sobre las respuestas de cada uno de los participantes es registrada y permitiría al profesor contar con la información diagnostica del grupo-clase en cuanto a saberes previos.

\subsection{Otras herramientas de MV disponibles.}

Existe software de licencia abierta como para construir un MV propio y las diversas herramientas de software que permitan construir objetos como los pulsadores para responder, utilizados en el ejemplo anterior. Luego de una selección se considera apta la tecnología ofrecida en Licencia Pública del originalmente llamado Project DarkStar y su última versión Project RedDwarf(RedDwarf, 2012). Esta es una solución de middleware de código abierto para el desarrollo del lado del servidor. En la actualidad se puede descargar el código fuente e iniciar un desarrollo independiente debiendo seguir con la licencia Open Source.

\subsection{Uso de Moodle como complemento del MV.}

En conjunto con las actividades a realizar en la plataforma de MV, se prevé usar Moodle como LMS para soportar la información de los asistentes, su usuario, contraseña, privilegios de acceso a los contenidos, provisión de material de estudio, seguimiento de actividades, resultado de las evaluaciones diagnósticas individuales, realización de actividades colaborativas convencionales, resultados individuales de los test que soporta Moodle.

La gestión de las actividades a realizar en cumplimiento de los requerimientos, acciones y recomendaciones, podrá ser soportada con un modelo de datos propio con tablas creadas conjuntamente con las tablas de Moodle, distinguida con una sigla al inicio del nombre de cada una, PTA. Esto requiere la construcción de formularios de acuerdo a las plantillas provistas por Moodle, para registrar el diagnostico del grupo clase ya que deberá llevarse registro de los datos de conformación del grupo, los integrantes de los mismos, las observaciones hechas y los resultados de las actividades realizadas por cada uno de los integrantes, como en la del ejemplo del concurso de preguntas y respuestas para obtener los conocimientos previos. 
Se necesitarán también tablas que almacenen la información de perfil de los participantes, las respuestas de cada estudiante con la opción elegida y las respuestas redactadas que las complementan, con la posibilidad de que el estudiante pueda acceder a todas sus respuestas pasadas y así poder corregir, mejorando los resultados del aprendizaje soportado por esta metodología.

También se colectará información adicional en tablas que ayuden a soportar la información de un perfil de desempeño, que permita analizar el rendimiento académico logrando así estimaciones previas (Pérez Cota, 2011). Estas estimaciones permitirán al docente actuar preventivamente conociendo las características del grupo-clase, realizando tareas de ajuste, administrando contenidos opcionales y extra, y otras estrategias que permitan mejorar los resultados de las actividades de enseñanza y aprendizaje con el soporte de la plataforma LMS y MV.

\section{Experiencia en Terreno}

Para la prueba inicial del concepto vinculado a complementar una clase presencial con una actividad en SL se llevó a cabo en Junio de 2009 durante la escuela de verano de la University of Applied Sciences of Mikkeli en Finlandia, una actividad en una clase del curso Human Interface Design dictada por el Dr. Manuel Pérez Cota de la Universidad de Vigo (España). El curso estaba conformado por estudiantes de diversos orígenes, de Alemania, China, España, Finlandia y Rusia. El instructor virtual estaba en Córdoba, Argentina y los guiaría por un lugar a partir del cual se cumplirían algunas actividades, como por ejemplo ver un video tutorial acerca de cómo realizar los primeros movimientos en SL.

La consigna fue que entraran al sitio www.temuestrocomo.com.ar (tmc, 2009) y desde el aula virtual de ese sitio, basada en Moodle, ingresaran a un curso. Allí se encontrarían con una lectura breve de instrucciones en Ingles y con un link a Second Life, al cual ingresarían con una identificación creada ad-hoc. Incluso se mencionó la posibilidad de que si alguno tuviera ya un usuario que lo aplicase y luego se trasladara al lugar del encuentro.

Durante el desarrollo de la actividad, se observó que de los quince estudiantes cinco poseían una cuenta en SL lo que hablaba de familiaridad con el recurso a introducir en la clase y con sus avatares aportaron colaborativamente algunas habilidades del entorno y de su mundo, como artículos de su inventario que compartieron con los compañeros, y en tomar la iniciativa en el uso del chat, compartiendo algunas instrucciones para realizar vuelos o movimientos especiales.

El profesor a cargo del curso presencial evaluó la actividad con los siguientes comentarios:

El único aspecto negativo de la experiencia es que hubo problemas con la 
conexión en momentos, congelando la acción de todos los participantes, atribuibles al comienzo al tráfico generado con la interacción de 15 estudiantes y el profesor en el aula de Mikkeli, usando la misma conexión a Internet, compartida con toda la Universidad.

Los aspectos positivos de la experiencia fueron los siguientes:

- Permitió una interacción desconocida hasta el momento por los estudiantes para un curso presencial.

- Les permitió ver que es posible, a distancia, llevar a cabo clases de temas simples y complejos.

- Les permitió ver que es posible, con los avatares, entender el funcionamiento de un aula virtual.

- Les permitió ver la importancia del buen diseño de un interfaz

- Les permitió ver que la investigación va, incluso, por delante de lo que pueden ver en la televisión

- Les permitió interactuar y trabajar con personas que son de ambientes culturales, idiomáticos, ambientales, completamente diferentes a los suyos

El instructor remoto, ubicado en Córdoba, Argentina, pudo ver otros aspectos adicionales, como que el profesor en Finlandia tuvo una actuación espontánea de observación y control de los asistentes, mientras que el instructor remoto hacia las veces de anfitrión en el mundo virtual.

Esta experiencia fue tomada como base para el diseño de actividades más significativas para la comprensión de materias corrientes de las carreras universitarias. Para esto fueron tomados en cuenta los requerimientos didácticos estudiados para poder crear una actividad que permitiera determinar los conocimientos previos (Ausubel, 1983).

La idea base para esta nueva actividad fue motivar a los asistentes a la materia a que formulen sin vergüenza ni timidez, lo que saben, poco o mucho, sobre el tema a dictarse, estimulando a que respondan a preguntas en la actividad descripta en el punto 4.2.

A esta combinación de didáctica y entretenimiento, la tecnología tiene mucho que aportar con desarrollo de software, diseño de interfaz y potencia de infraestructura para soportar muchos asistentes virtuales a la actividad con la mayor continuidad de servicio.

\section{Conclusiones}

La convergencia de Estrategias didácticas de avanzada como las actividades adaptativas de Piaget, el aprendizaje como descubrimiento de Bruner, determinar los conocimientos previos de Ausubel, el medio social de Vigotsky reunidos en una experiencia novedosa de Blended Learning como marco de trabajo, y el 
aporte de la Ingeniería de Software para el desarrollo de aplicaciones con normas de calidad y testeo permitirá un avance significativo en el logro del aprendizaje de los estudiantes, los nativos digitales de Prensky que pueblan las Escuelas y Universidades en la actualidad.

El desarrollo tecnológico para esta experiencia está en el servidor www. temuestrocomo.com.ar que permitió dar las instrucciones y hacer llegar a los participantes a un lugar específico de SL, con unos usuarios preparados y en el sitio de SL que es una infraestructura compleja con un gran despliegue en sus servidores dedicados para ofrecer la experiencia de inmersión en un software de MV.

Se plantea así la posibilidad de vincular Blended Learning con Mundos Virtuales para soportar encuentros sincrónicos, con la riqueza de recursos disponibles a fin de desarrollar actividades de aprendizaje significativo en un entorno símil a un video juego, sumando valor y se sugiere que sea bajo el término "Blended Learning 2.0". 


\section{Bibliografía}

Ausubel, David et al (1983) "Psicología Educativa: Un punto de vista cognoscitivo" México, 1983. Ed.TRILLAS.

Bruner, Jerome. (1997) "La educación puerta de la cultura" Madrid, 1997. Ed. Visor. Colección Aprendizaje ${ }^{\circ} 125$.

Campazzo E. Et al (2011) - DE LA PRESENCIALIDAD A LA INTERACCION VIRTUAL 3D - Revista Calidad en la Educación Superior -Universidad Estatal a Distancia ISSN 1659-4703 Costa Rica.

Carretero, Mario (2009) "Constructivismo y Educación" - 1 Edición. Buenos Aires: Paidos. Pag. 24

La Fuente, J V, et al (2012) "Entorno Virtual de Aprendizaje Evalpa" Revista Ibero Americana de educación Monografico.60 Septiembre-Diciembre 2012 www.rieoei.org/rie60.pdf - "Entornos virtuales de aprendizaje en Iberoamérica" Edit Centro de Estudios Universitarios CAEU Org. Estados Iberoamericanos para la educación, ciencia y cultura (OEI) ISSN 1022-6508 - visitado en abril 2013.

Lopes, C. V., Debeauvais, T., \& Valadares, A. (2012, September). RESTful Massively Multi-User Virtual Environments: A Feasibility Study. In Games Innovation Conference (IGIC), 2012 IEEE International (pp. 1-4). IEEE.

LSL, Linden Scripting Language, (2013) http://wiki.secondlife.com/wiki/LSL Tutoriales visitado en Julio del 2013

Open Wonderland http://openwonderland.org/ visitado en Abril del 2012.

Pérez Cota, Manuel et al (2012). Requerimientos de la didáctica para una estrategia de aplicación de mundos virtuales para educación. In XIV Workshop de Investigadores en Ciencias de la Computación.

Pérez Cota, Manuel et al (2011) “Utilizando Perfiles de Desempeño Para Prever el Rendimiento Académico" Capitulo de "La Tecnología Educativa al Servicio de la Educación Tecnológica" Buenos Aires, 2010. Ed. edUTecNe. Paginas 172 a 200

Piscitelli, A. (2009). Nativos digitales. Buenos Aires: Santillana.

Prensky, Marc (2006) "Mom don't bother me, I'm learning" http://www. marcprensky.com/ visitado en enero 2012

RedDwarf(2012)http://en.wikipedia.org/wiki/RedDwarf_ServerVisitado Febrero 2012 Second Life (2013) http://secondlife.com/whatis/faq.php\#02. - visitado en Febrero 2013 
Souto, Marta (1999) Grupos y dispositivos de formación. Colección Formación de Formadores. Buenos Aires. Ediciones Novedades Educativas y Facultad de Filosofía y Letras, UBA.

Tmc (2009) http://www.temuestrocomo.com.ar/joomla/index.php?limitstart=5 vínculo donde se describe los datos del encuentro con los estudiantes del curso Efist - Human Interface Design Course de la Universidad de Mikkeli

Vigotsky, Lev (1988) "El desarrollo de los procesos psicológicos superiores" México Editorial Crítica Grupo Editorial Grijalbo. 